\title{
New Sequential Copolymers and Networks by a Combination of Techniques
}

\author{
Joseph P. KENNEDY \\ Institute of Polymer Science, The University of Akron, \\ Akron, Ohio 44325, U.S.A.
}

(Received August 20, 1984)

\begin{abstract}
Research into the mechanism of the inifer process led to perfectly endfunctionalized (telechelic) linear or three-arm star polyisobutylenes (PIB)s. Recently a semicontinuous technique has been developed and is now routinely employed for the synthesis of these materials. The ingredients are fed into a stirred reactor and by controlling their input rates PIB telechelics with virtually theoretical $M_{n}$ and $M_{w} / M_{n}$ are harvested. The first intermediates that form are $t$-chlorine telechelic PIBs which can then be quantitatively converted to other well-defined telechelics, e.g., PIBs carrying olefin, alcohol, phenol, etc. end groups. PIB diols and triols are now available on a semicommercial scale in various molecular weights. Telechelic PIBs have been used for the preparation of new block copolymers and/or networks by a variety of techniques. For example, a combination of polymerization and coupling techniques have been employed for the synthesis of a new class of di- and triblock copolymers consisting of PIB and polyethylene glycol sequences. Thus phenol-ended PIBs were reacted with a slight stoichiometric excess of diisocyanates, and the resulting isocyanate-capped prepolymers were then reacted with commercially available polyethylene glycols. These hydrophobic/hydrophilic (amphiphilic) materials exhibit some unusual dilute solution properties.

KEY WORDS Inifer Process / Telechelic Polyisobutylenes / Phenol-Ended Polyisobutylenes / Polyisobutylene Blocks /
\end{abstract}

The discovery of inifer system ${ }^{1,2}$ resulted in the convenient synthesis of well-defined end-reactive (telechelic) PIBs and subsequently led to a large variety of intermediates useful for the preparation of PIB-based polyurethanes, epoxy resins, etc. ${ }^{3}$ These new materials contain PIB sequences and as a result exhibit increased oxidative chemical and environmental resistance, hydrolytic stability, and barrier properties. ${ }^{4}$

The first intermediates obtained by the inifer technique are tert-chlorine telechelic PIBs. ${ }^{5,6}$ Depending on the specific inifer used (minifer, binifer, or trinifer) linear or three-arm star prepolymers may be prepared. The linear telechelic PIB may carry $\alpha$-phenyl- $\omega$-tert-chloro end groups (asymmetric telechelic) or $\alpha, \omega$-di-tert-chloro end groups (symmetric telechelic); the three-arm star species carries three tert-chloro termini ${ }^{7}$ :

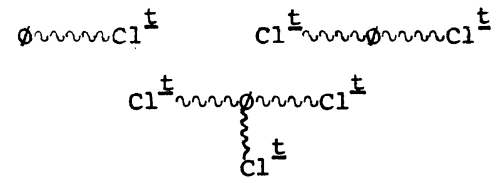

Insight into the mechanism of the various inifer systems led to molecular weight control. ${ }^{8}$ Thus the $M_{n}$ of tert-chlorine telechelic PIB can be controlled in the $\sim 1000$ to $\sim 10,000$ dalton range. Molecular weight control of course is possible to much higher levels, however, direct end group quantitation becomes increasingly difficult with higher molecular weight materials.

Coupled with these mechanistic/kinetic investigations organic chemical methods have been developed that led to the quantitative conversion of the tert-chloro end groups to other useful functions, e.g., olefin, epoxy, alcohol, phenol. $^{7}$ These derivatives have then been employed for the preparation of unique new engineering materials. ${ }^{7}$ 


\section{J. P. KENNEDY}

In the course of our detailed investigations of the mechanism of inifer systems we have found that sometimes the low molecular weight materials $\left(M_{n}\right.$ from $\sim 500$ to $\sim 6,000$ ) may carry "unfired" or "once fired" end groups, for example, in the binifer system:

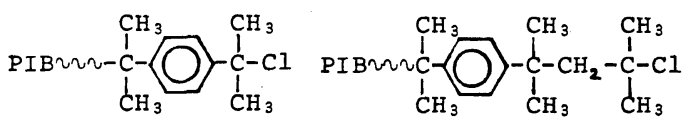

unfired end group

once-fired end group

The presence of these end groups in the final product leads to a broadening of the molecular weight distribution (from $M_{w} / M_{n}=1.5$ to $\sim 2.0$ ). The theoretical molecular weight dispersity ratios $M_{w} / M_{n}$ were shown to be 1.5 for the linear twoended telechelics, ${ }^{8}$ and 1.33 for the three-arm stars, ${ }^{9}$ prepared at low monomer conversions in batch systems.

While the number average end group functionality $\bar{F}_{n}$ of the product containing unfired or oncefired end groups is $\bar{F}_{n}=2.0$ (for linear) or $\bar{F}_{n}=3.0$ (for three-arm star), the reactivity of the end groups may be somewhat different from the main product:<smiles>CCC(C)(C)CC(C)(C)CC(C)(C)Cl</smiles>

French scientists have recently shown that linear oligoisobutylenes may contain a significant amount of once-fired end groups. ${ }^{10}$

Another potential difficulty that may occasionally occur is due to insufficient reactor control i.e., unsatisfactory stirring, insufficient temperature control, too high conversions; this may result in broader than theoretical or even multimodal molecular weight distributions.

Recently a semicontinuous polymerization method has been developed to overcome these potentially disturbing features. ${ }^{9}$ Under semicontinuous conditions perfectly symmetrical end-reactive prepolymers can be obtained with close to theoretical molecular weight dispersities, i.e., $M_{w} / M_{n}=1.5$ for linear and 1.33 for three-arm star products, even at complete monomer conversions. The first part of this presentation concerns an out-line of this semicontinuous method and the results obtained by the use of it.

The second part of this lecture describes the synthesis of a new family of amphiphilic materials comprising of hydrophobic PIB segments linked to hydrophilic poly(ethylene glycol) PEG segments. ${ }^{11}$

Sequential copolymers comprising of hydrophobic/hydrophilic blocks are of great practical interest for a variety of applications e.g., compatibilizing agents, emulsifying aids, delayed drug delivery systems, in cosmetics, in agriculture, in water treatment. Investigations focusing on amphiphilic polymers in which the hydrophobic moiety was PIB, have resulted in a new block copolymer poly(ethyleneimine- $b$-isobutylene $)^{12}$ and amphiphilic networks of PIB and for example $\operatorname{poly}(N$ vinyl pyrrolidone). ${ }^{13}$

The ready availability of various well-defined telechelic PIBs prompted us to extend these investigations to the synthesis of PIB/PEG amphiphilics and to study some of their dilute solution properties.

\section{EXPERIMENTAL}

Semicontinuous runs were carried out under nitrogen in three-neck flasks. The reactor contained cooled dilute $\mathrm{BCl}_{3} /$ solvent charges into which were continuously introduced precooled inifer/ isobutylene/solvent feeds. Binifer experiments were performed at $-80^{\circ}$ with $\mathrm{CH}_{2} \mathrm{Cl} / n-\mathrm{C}_{6} \mathrm{H}_{14}(80 / 20$ $\mathrm{v} / \mathrm{v}$ ), and trinifer runs at $-40^{\circ}$ with $\mathrm{CH}_{3} \mathrm{Cl}$. In batch runs $\mathrm{BCl}_{3}$ solutions were rapidly added to inifer/ isobutylene/solvent charges. The compositions of the final charges in the semicontinuous and batch runs were identical. Further experimental details are given in ref 9.

The PIB/PEG amphiphilics were prepared by reacting PIB monols or diols with a slight stoichiometric excess of 2,4-tolylene-diisocyanate TDI, and coupling the isocyanate capped products with various grades (molecular weights) of polyethylene glycols. The block copolymer products were purified by column chromatography and the dilute solution properties were studied by viscometry and surface tension measurements. Further experimental data are given in ref 11 .

\section{RESULTS AND DISCUSSION}

I. Semicontinuous Inifer Systems

A) Kinetic Considerations.

The following elementary reactions describe the 
polymerization of isobutylene (M) by the inifer (I)/

$\mathrm{BCl}_{3}$ system:

Ion generation

$$
\mathrm{I}+\mathrm{BCl}_{3} \frac{k_{\mathrm{i}}}{\underset{k_{-\mathrm{i}}}{\rightleftharpoons}} \mathrm{I}^{\oplus} \mathrm{BCl}_{4}^{\ominus}
$$

Cationation

$$
\stackrel{\mathrm{I}^{\oplus}}{\longleftarrow} \mathrm{M} \stackrel{k_{\mathrm{c}}}{\longleftarrow} \mathrm{M}_{n}^{\oplus}
$$

Propagation

$$
\mathrm{M}_{n}^{\oplus}+\mathrm{M} \stackrel{k_{\mathrm{p}}}{\longrightarrow} \mathrm{M}_{n+1}^{\oplus}
$$

Chain transfer to inifer

$$
\mathrm{M}_{n}{ }^{\oplus}+\stackrel{k_{\mathrm{tr}, \mathrm{I}}}{\longrightarrow} \mathrm{I}^{\oplus}+\mathrm{M}_{n}
$$

Termination

$$
\mathrm{M}_{n}^{\oplus} \mathrm{BCl}_{4}^{\ominus} \stackrel{k_{\mathrm{t}}}{\longrightarrow} \mathrm{M}_{n}+\mathrm{BCl}_{3}
$$

where $\mathbf{M}_{n}$ is polymer, $\mathrm{I}^{\oplus}$ and $\mathrm{M}_{n}{ }^{\oplus}$ are inifer and polymer cations, and the rate constants have their usual notations. Chain transfer to $M$ was shown to be absent up to $-20^{\circ}$ in this system. ${ }^{14,15}$

It has been shown that when $k_{\mathrm{tr}, \mathrm{I}}[\mathrm{I}]+k_{\mathrm{t}} \ll k_{\mathrm{p}}[\mathrm{M}]$, the polymer formed will exhibit $M_{w} / M_{n}=1.5$ in the binifer system and $M_{w} / M_{n}=1.33$ in the trinifer system. ${ }^{9}$

The number average degree of polymerization in inifer systems is governed by $[\mathrm{I}]$ and $[\mathrm{M}]^{8}$ :

$$
\overline{D P}_{n}=\frac{k_{\mathrm{p}}[\mathrm{M}]}{k_{\mathrm{tr}, \mathrm{L}}[\mathrm{I}]+k_{\mathrm{t}}}
$$

Obviously, in conventional batch polymerization systems both $[\mathrm{M}]$ and $[\mathrm{I}]$ decrease with time so that $\overline{D P}_{n}$ may decrease with progressing monomer conversion which in turn would result in broadened molecular weight distributions, i.e., $M_{w} / M_{n}$ would become larger than the theoretical value.

Thus polymers with close to theoretical $M_{w} / M_{n}$ could be prepared even at high conversions provided $[\mathrm{M}]$ and $[\mathrm{I}]$ remain constant during the run. This requirement can be met by continuously adding at a constant slow rate a monomer/inifer feed to a $\mathrm{BCl}_{3}$ coinitiator charge, making certain that the rate of the mixed monomer/inifer addition and that of monomer/inifer consumption remain equal during the polymerization.

Based on eq 1 to 5

$$
\begin{gathered}
\frac{\mathrm{d}[\mathrm{M}]}{\mathrm{d} t}=A_{\mathrm{M}}-k_{\mathrm{c}}\left[\mathrm{I}^{\oplus}\right][\mathrm{M}]-k_{\mathrm{p}}\left[\mathrm{M}_{n}^{\oplus}\right][\mathrm{M}] \\
\frac{\mathrm{d}[\mathrm{I}]}{\mathrm{d} t}=A_{\mathrm{I}}-k_{\mathrm{i}}[\mathrm{I}]\left[\mathrm{BCl}_{3}\right]-k_{\mathrm{tr}, \mathrm{I}}\left[\mathrm{M}^{\oplus}\right][\mathrm{I}]+k_{-\mathrm{i}}\left[\mathrm{I}^{\oplus}\right]
\end{gathered}
$$

where $A_{\mathrm{M}}$ and $A_{\mathrm{I}}$ are the addition rates of $\mathrm{M}$ and I, respectively. Under stationary conditions

$$
\frac{\mathrm{d}[\mathrm{M}]}{\mathrm{d} t}=0 \quad \text { and } \quad \frac{\mathrm{d}[\mathrm{I}]}{\mathrm{d} t}=0
$$

so that

$$
A_{\mathrm{M}}=k_{\mathrm{p}}\left[\mathrm{M}_{n}^{\oplus}\right][\mathrm{M}]+k_{\mathrm{c}}\left[\mathrm{I}^{\oplus}\right][\mathrm{M}]
$$

and it can be shown (9) that

$$
A_{\mathrm{I}}=k_{\mathrm{tr}, \mathrm{I}}\left[\mathrm{M}_{n}^{\oplus}\right][\mathrm{I}]+k_{\mathrm{t}}\left[\mathrm{M}_{n}^{\oplus}\right]
$$

In view of eq 2 to 5

$$
\overline{D P}_{n}=\frac{k_{\mathrm{p}}\left[\mathrm{M}_{n}{ }^{\oplus}\right][\mathrm{M}]+k_{\mathrm{c}}\left[\mathrm{I}^{\oplus}\right][\mathrm{M}]}{k_{\mathrm{tr}, \mathrm{I}}\left[\mathrm{M}_{n}{ }^{\oplus}\right][\mathrm{I}]+k_{\mathrm{t}}\left[\mathrm{M}_{n}{ }^{\oplus}\right]}
$$

and by substituting eq 10 and 11 we obtain

$$
\overline{D P}_{n}=\frac{A_{\mathrm{M}}}{A_{\mathrm{I}}}
$$

Since the feed contains both the monomer and inifer in a common solvent:

$$
\overline{D P}_{n}=\left(\frac{[\mathrm{M}]}{[\mathrm{I}]}\right)_{\text {feed }}
$$

The stationary monomer and inifer concentration can be calculated ${ }^{9}$ :

$$
[\mathrm{M}]=\frac{1}{2} B+\left[\frac{1}{4} B^{2}+\left(B+\frac{k_{-\mathrm{i}}}{k_{\mathrm{c}}}\right) \dot{C}\right]^{1 / 2}
$$

where

$$
B=\frac{k_{\mathrm{t}}}{k_{\mathrm{p}}}\left(\frac{A_{\mathrm{M}}}{A_{\mathrm{I}}}-1\right) \quad \text { and } C=\frac{k_{\mathrm{tr}, \mathrm{I}}}{k_{\mathrm{p}}} \frac{\left(A_{\mathrm{M}}-A_{\mathrm{I}}\right)}{k_{\mathrm{i}}\left[\mathrm{BCl}_{3}\right]}
$$

and

$$
\begin{aligned}
{[\mathrm{I}]=} & \frac{1}{2} \frac{k_{\mathrm{t}}}{k_{\mathrm{tr}, \mathrm{I}}}+\left\{\frac{1}{4}\left(\frac{k_{\mathrm{t}}}{k_{\mathrm{tr}, \mathrm{I}}}\right)^{2}\right. \\
& \left.+\left(B \frac{k_{-\mathrm{i}}}{k_{\mathrm{c}}}\right) \frac{k_{\mathrm{p}}}{k_{\mathrm{tr}, \mathrm{I}}} \frac{A_{\mathrm{I}}{ }^{2}}{A_{\mathrm{M}}-A_{\mathrm{I}}} \frac{1}{k_{\mathrm{i}}\left[\mathrm{BCl}_{3}\right]}\right\}^{1 / 2}
\end{aligned}
$$

The value of $[\mathrm{M}]$ depends on both the relative and absolute addition rates $A_{\mathrm{M}}$ and $A_{\mathrm{l}}$, i.e., at a given $[\mathrm{M}] /[\mathrm{I}]$ in the feed, [M] would increase with increasing addition rate. Thus the addition rate should be 


\section{J. P. KENNEDY}

sufficiently low to achieve stationary conditions rapidly. Otherwise monomer/inifer may accumulate in the reactor which may lead to nonstationary conditions which in turn would approach conventional batch polymerization conditions.

B) Experimental Results.

A series of parallel experiments have been carried out: One was a semicontinuous run and the "control" was a conventional batch experiment. The final concentrations were the same in both runs.
Talbe I shows the results.

Polymers with $M_{w} / M_{n}$ close to theoretical values have been obtained in the semicontinuous experiments. Dispersities were much broader in batch runs and GPC traces exhibited multimodality.

The products have been analyzed by ${ }^{1} \mathrm{H}$ NMR spectroscopy. Confirming our earlier observations and those of French scientists, ${ }^{10}$ the products obtained with binifer do not contain unfired end groups (see above) but may contain once-fired end

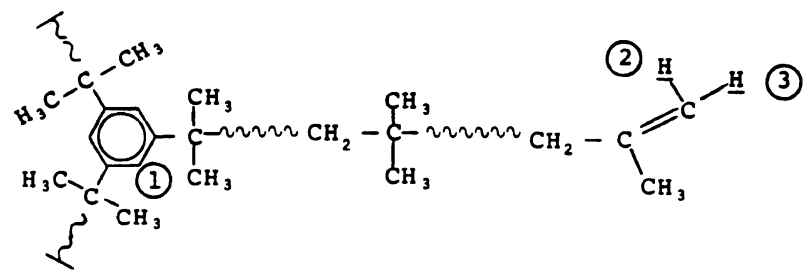

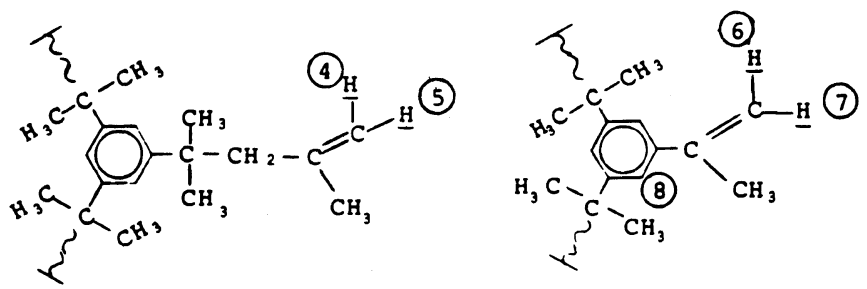

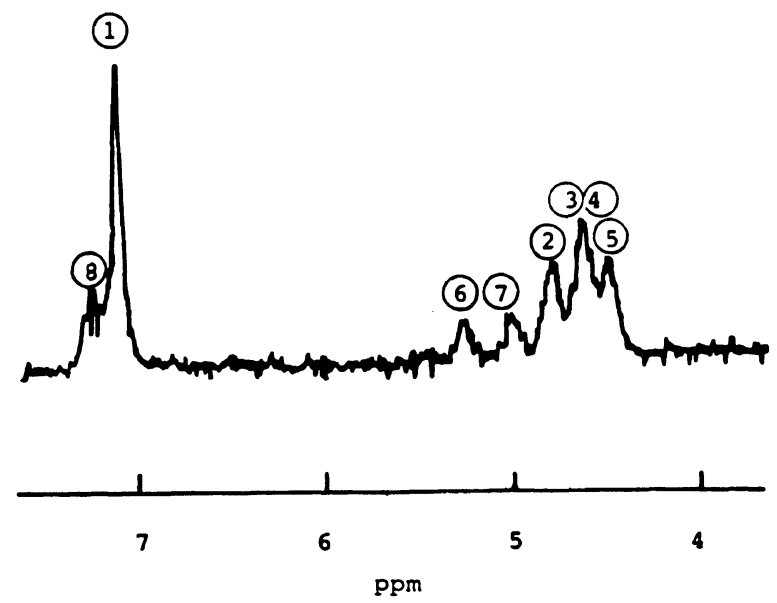

(1) : $7.15 \mathrm{ppm},(2): 4.8 \mathrm{ppm},($ (3) $: 4.65 \mathrm{ppm}$, (4): $4.65 \mathrm{ppm}$,
(5) $: 4.5 \mathrm{ppm}$, (6) $: 5.30 \mathrm{ppm}$, (7) $: 5.00 \mathrm{ppm}$, (8): $7.25 \mathrm{ppm}$

Figure 1. ${ }^{1} \mathrm{H}$ NMR spectrum of product $4 \mathrm{~b}$ of Table I obtained after dehydrochlorination (for assignments see ref 7). 
Sequential Copolymers and Networks

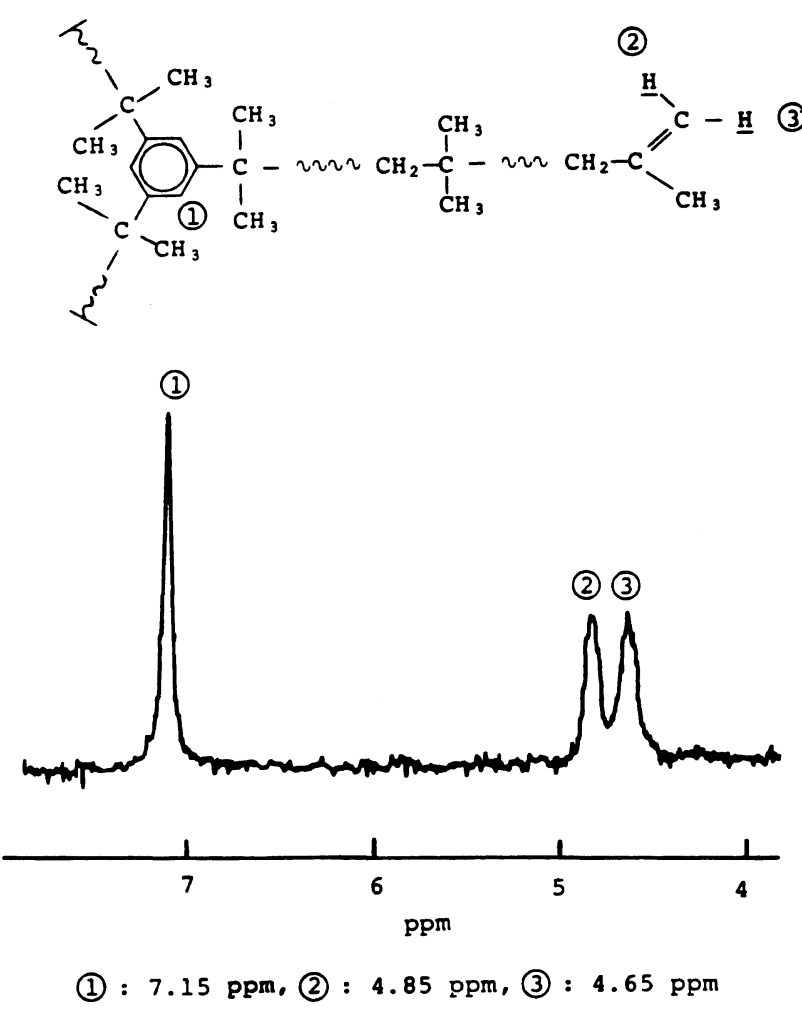

Figure 2. ${ }^{1} \mathrm{H}$ NMR spectrum of product $4 \mathrm{a}$ of Table I obtained after dehydrochlorination (for assignments see ref 7).

groups.

The prepolymers obtained with trinifer in batch runs contain both unfired and once-fired end groups. In contrast, polymers prepared using the same concentration of ingredients but under semicontinuous conditions, are virtually free of unfired or once-fired end groups. Inspection of representative NMR spectra shown in Figures 1 and 2 illustrates the findings. (To facilitate NMR interpretation the tert-chloro end groups have been quantitatively converted to exo olefin end groups. ${ }^{16}$ )

Molecular weight dispersity data shown in Table I corroborate these observations. Products containing unfired or once-fired end groups would not yield theoretical $M_{w} / M_{n}$ values even if $[\mathrm{M}] /[\mathrm{I}]$ would remain constant during the run. Or, to state this another way: Products with theoretical $M_{w} / M_{w}$ values must be devoid of species carrying unfired or once-fired end groups. Thus the close to theoretical $M_{w} / M_{n}$ values obtained in trinifer runs indicate the virtual absence of unfired and/or once-fired end groups.
II. Synthesis and Characterization of Polyiso- butylene/Poly(ethylene glycol) Block Copoly- mers

Amphiphilic block or graft copolymers are extensively used for a variety of applications e.g., emulsifying agents, foaming or anti-foaming products, and the polyethylene glycol PEG segment is often part of these materials as the hydrophilic moiety. Since the commercial introduction of poly(propylene oxide- $b$-ethylene oxide) nonionic surfactants many polyols have been studied, however, the hydrophobic moieties were usually propylene oxide or low molecular weight $\left(\mathrm{C}_{8}-\mathrm{C}_{12}\right)$ hydrocarbons. High molecular weight hydrocarbons are commonly not used as the hydrophobic component and PIB-containing amphiphilic block copolymers are virtually unknown. Attempts have been made to prepare poly(isobutylene- $b$-ethylene oxide) block copolymers by blocking ethylene oxide 


\section{J. P. KENNEDY}

Table I. Comparison of semicontinuous and conventional polymerization techniques for the preparation of telechelic polyisobutylenes by binifer and trinifer

\begin{tabular}{|c|c|c|c|c|c|c|c|}
\hline & \multirow{2}{*}{$\begin{array}{c}\mathrm{BCl}_{3} \\
\text { in initial charge }\end{array}$} & \multirow{3}{*}{$\mathrm{IB}^{\mathrm{a}}$} & \multirow{3}{*}{$\begin{array}{c}\begin{array}{c}\text { Inifer } \\
\text { in feed }\end{array} \\
M\end{array}$} & \multicolumn{2}{|c|}{ Feed } & \multirow{3}{*}{$M_{n}$} & \multirow{3}{*}{$M_{w} / M_{r}$} \\
\hline & & & & Rate & Time & & \\
\hline & $M \times 10$ & & & $\mathrm{ml} \mathrm{min}^{-1}$ & $\min$ & & \\
\hline & \multicolumn{7}{|c|}{ Semicontinuous binifer runs: $-80^{\circ} \mathrm{C}, \mathrm{CH}_{3} \mathrm{Cl} / n$-hexane solvent mixture $(80 / 20, \mathrm{v} / \mathrm{v})$} \\
\hline $1 \mathrm{a}$ & 1.9 & 3.7 & $2.1 \times 10^{-1}$ & 11 & 6 & 980 & 2.0 \\
\hline $2 \mathrm{a}$ & 1.6 & 5.0 & $1.5 \times 10^{-1}$ & 8 & 92 & 2000 & 1.8 \\
\hline \multirow[t]{2}{*}{$3 \mathrm{a}$} & 4.7 & 3.3 & $6.5 \times 10^{-1}$ & 10 & 6 & 2900 & 1.8 \\
\hline & \multicolumn{5}{|c|}{ Semicontinuous trinifer runs: $-40^{\circ} \mathrm{C}, \mathrm{CH}_{3} \mathrm{Cl}$} & & \\
\hline $4 \mathrm{a}$ & 4.7 & 6.4 & $1.7 \times 10^{-1}$ & 15 & 2.5 & 2500 & 1.4 \\
\hline $5 a$ & 4.7 & 4.0 & $6.8 \times 10^{-2}$ & 21 & 2.1 & 3900 & 1.5 \\
\hline $6 a$ & 4.7 & 6.0 & $5.2 \times 10^{-2}$ & 7 & 8 & 6700 & 1.3 \\
\hline \multirow[t]{3}{*}{$7 a$} & 4.7 & 6.0 & $4.1 \times 10^{-2}$ & 11 & 5.1 & 8200 & 1.3 \\
\hline & $\mathrm{BCl}_{3} M \times 10$ & $\mathrm{IB}^{\mathrm{a}} M$ & Binifer $M$ & & & $M_{n}$ & $M_{w} / M_{n}$ \\
\hline & \multicolumn{7}{|c|}{ Conventional binifer runs: $-80^{\circ} \mathrm{C}, \mathrm{CH}_{3} \mathrm{Cl} / n$-hexane solvent mixture $(80 / 20, \mathrm{v} / \mathrm{v})$} \\
\hline $1 \mathrm{~b}$ & 1.6 & 0.8 & $4.5 \times 10^{-2}$ & & & 900 & 3.6 \\
\hline \multirow[t]{2}{*}{$3 b$} & 3.1 & 1.3 & $2.6 \times 10^{-2}$ & & & 3200 & 3.0 \\
\hline & \multicolumn{5}{|c|}{ Conventional trinifer runs: $-40^{\circ} \mathrm{C}, \mathrm{CH}_{3} \mathrm{Cl}$ solvent } & & \\
\hline $4 b$ & 3.3 & 1.7 & $4.6 \times 10^{-1}$ & & & b & \\
\hline $5 b$ & 3.2 & 1.2 & $2.0 \times 10^{-1}$ & & & b & \\
\hline $5 b$ & 2.9 & 2.1 & $1.8 \times 10^{-1}$ & & & b & \\
\hline
\end{tabular}

a Monomer conversion was $100 \%$.

b Multimodal molecular weight distribution, see Figure 1.

from chlorine-telechelic PIB, however, the synthetic procedure was found to be cumbersome. ${ }^{17}$ Thus it appeared of interest to search for a convenient efficient route for the synthesis of PIB/PEG block copolymers.

The ready availability of various well-defined telechelic PIBs prompted us to use these as starting materials for the preparation of PIB/PEG block copolymers. A series of experiments have been designed for the synthesis of linear PIBs carrying one and/or two $p$-phenol termini, ${ }^{18}$ convert these to isocyanate termini (capping) and subsequently to couple these with PEG blocks carrying one hydroxyl germinus. Tolylene-2,4-diisocyanate TDI and diphenylmethane-4,4-diisocyanate MDI were examined as isocyanate capping agents and it was found that TDI gives clear capping (no extension), probably because of the dissimilar reactivities of the -NCO groups in this molecule. The following set of equations serves to visualize the conversions employed:

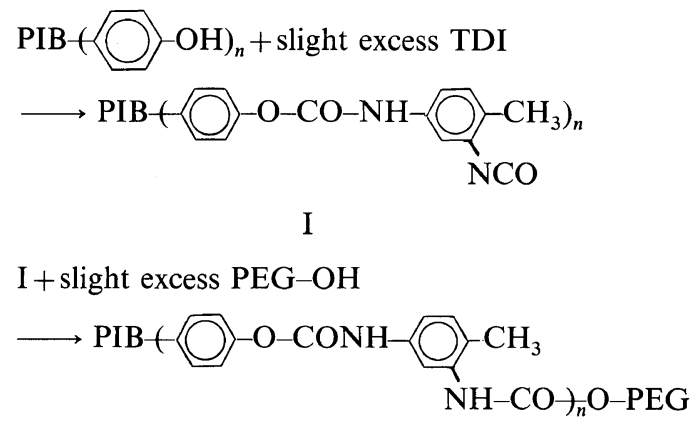

where $n=1$ or 2 . Thus by the use of PIB- $-\mathrm{OH}$ we obtained PIB/PEG diblocks, whereas with HO-PIB- $\bigcirc-\mathrm{OH}$ the end products were $\mathrm{PEG} /$ PIB/PEG triblock copolymers. The connecting link between the hydrophilic PEG and hydrophobic PIB segments was the aromatic diurethane unit (see above).

The separation of block copolymers from the homopolymer constituents and by products was an 


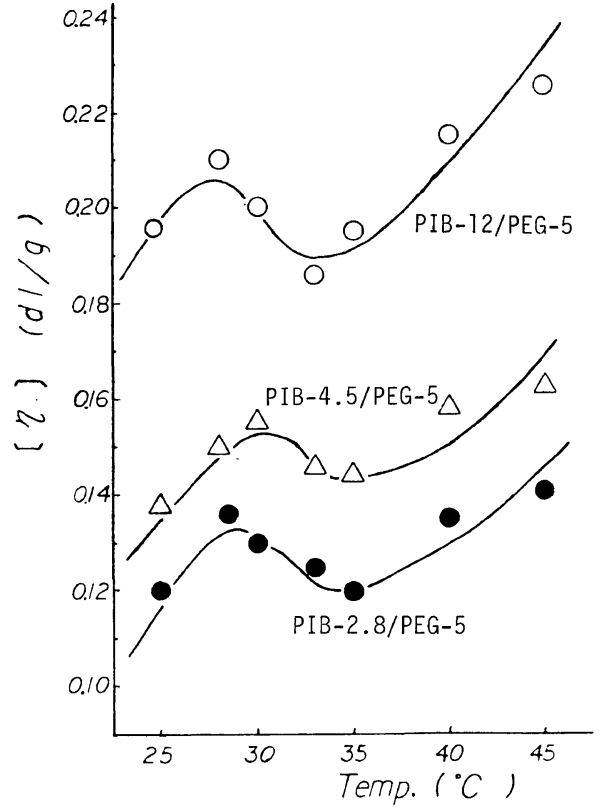

Figure 3. Temperature dependence of intrinsic viscosity of PIB/PEG block copolymers (in toluene). The numbers in the figure indicate $M_{n} \times 10^{3}$. For example, PIB-12 $=$ PIB of $M_{n} 12,000$

extremely demanding and cumbersome undertaking, nonetheless, it was accomplished by column chromatography (silica packing) using benzenemethanol mixtures as the eluent. ${ }^{11}$ Blocking efficiencies were a minimum of $75-85 \%$.

The thermal behavior (DSC), interfacial surface characteristics and dilution solution properties (viscosities) of PIB/PEG di- and tri-block copolymers have been investigated. As expected, DSC showed a glass transition exotherm at low temperatures $\left(T_{\mathrm{g}} \sim-70^{\circ} \mathrm{C}\right)$ associated with the PIB moiety, and a melting exotherm. at higher temperatures $(\mathrm{mp}$ $\sim 53^{\circ} \mathrm{C}$ ) indicating the presence of crystalline PEG blocks. The interfacial surface tension of PIB/PEG block copolymers at benzene/water interfaces has been determined. A block copolymer comprising of a PIB block of $M_{n}=12,000$ and a PEG block of $M_{n}=5000$ exhibited a critical micelle concentration of $3.0 \times 10^{-5} \mathrm{~mol}^{-1}$ which is a reasonable value for a long chain nonionic surfactant. Turbidimetry in methanol $/ n$-hexane systems also indicated typical amphiphilic behavior.

The intrinsic viscosity of various PIB/PEG block copolymers as a function of temperature was in- vestigated in the 25 to $45^{\circ} \mathrm{C}$ range in toluene solvent. Figure 3 shows the findings obtained with three block copolymers. While the intrinsic viscosity of the homopolymers decreases monotonically with increasing temperatures (not shown), the block copolymers exhibit a characteristic discontinuous behavior. Thus the intrinsic viscosities increase with increasing temperatures in the 25-30 and 35$45^{\circ} \mathrm{C}$ ranges. Similarly, the dependence of intrinsic viscosity on polar/nonpolar solvent composition (i.e., in methanol $/ n$-hexane systems also showed a pattern characteristics of intrinsically immiscible amphiphilic block copolymers.

Acknowledgements. Financial support for this research has been provided by the National Science Foundation (Grant DMR-81-20964).

\section{REFERENCES}

1. J. P. Kennedy, R. A. Smith, and L. R. Ross, U.S. Patent 4,276,394 (1981).

2. J. P. Kennedy, U.S. Patent 4,316,973 (1982).

3. J. P. Kennedy and S. Guhaniyogi, U.S. Patent4, 429,099 (1984).

4. V. S. C. Chang and J. P. Kennedy, Polym. Bull., 8, 69 (1982).

5. J. P. Kennedy and R. A. Smith, J. Polym. Sci., Polym. Chem. Ed., 18, 1539 (1980).

6. J. P. Kennedy, L. R. Ross, J. E. Lackey, and O. Nuyken, Polym. Bull., 4, 67 (1981).

7. J. P. Kennedy, The Proceedings Robert A. Welch Conference Chemical Research, XXVI. Synth. Polym., Chap. III, 71-111 (1982).

8. A. Fehérvári, J. P. Kennedy, and F. Tüdös, $J$. Macromol. Sci., Chem., A15, 215 (1980).

9. R. Faust, A. Fehérvári, and J. P. Kennedy, A.C.S. Symposium Series, in press.

10. M. Tessier and E. Maréchal, Polym. Bull., 10, 152 (1983).

11. Y. Hongu, Ph. D. Disseration, The University of Akron, Akron, Ohio, 1985.

12. V. Percec, S. C. Guhaniyogi, J. P. Kennedy, and B. Iván, Polym. Bull., 8, 25 (1982).

13. B. Keszler and J. P. Kennedy, J. Macromol. Sci., Chem., A21(3), 319 (1984).

14. R. Santos, A. Fehérvári, and J. P. Kennedy, J. Polym. Sci., Polym. Chem. Ed., in press.

15. R. Santos, J. P. Kennedy, and W. Michael, Polym. Bull., 11, 261 (1984).

16. J. P. Kennedy, V. S. C. Chang, R. A. Smith, and B. Iván, Polym. Bull., 1, 575 (1979).

17. W. Francik, Ph. D. Dissertation, The University of Akron, Akron, Ohio 1983.

18. J. P. Kennedy, S. Guhaniyogi, and V. Percec, Polym. Bull., 8, 563 (1982). 